\title{
Efecto de la inclusión de harina de pisonay (Erythrina edulis) de tres edades de rebrote sobre las características productivas en cuyes (Cavia porcellus)
}

\author{
Effect of the inclusion of pisonay meal (Erythrina edulis) of three regrowth ages on \\ the productive characteristics in guinea pigs (Cavia porcellus)
}

\author{
Ludwing A. Cárdenas-Villanueva ${ }^{1,2,4}$, Ruth Ramos-Zuñiga ${ }^{3}$, José L. Huamán- \\ Gamarra $^{2}$, Eliseo Ramirez Mena ${ }^{2}$
}

\section{Resumen}

El objetivo del presente estudio fue evaluar el efecto de tres edades de rebrote y tres niveles de inclusión de harina de pisonay (Erythrina edulis) sobre el consumo y conversión alimenticia; la ganancia de peso, y el rendimiento de carcasa en el engorde de cuyes (Cavia porcellus). Se utilizó las hojas y los peciolos secados bajo sombra de árboles cosechados, y procesados mecánicamente a $2 \mathrm{~mm}$ de tamaño de partícula. Se utilizaron 120 cuyes machos mejorados, distribuidos en 10 tratamientos ( 3 edades de rebrote, tres niveles de inclusión de la harina y un grupo control con harina de alfalfa) durante 56 días. La ganancia de peso total y diaria, además de la conversión alimenticia tuvieron un comportamiento similar entre los tratamientos; el consumo del alimento fue menor con el tratamiento $8 \mathrm{M} 30 \%$ (54.0 g/día) con respecto al CA20\% (59.3 g/día) $(\mathrm{p}<0.05)$ y el rendimiento de carcasa fue mayor en $4 \mathrm{M} 20 \%(73.5 \%)$ en comparación con la dieta control $(71.1 \%)(\mathrm{p}<0.05)$. El consumo del alimento (60.3 g/día) como la conversión alimen-

${ }^{1}$ Laboratorio de Farmacología, Toxicología y Bioquímica Veterinaria, Universidad Nacional Micaela Bastidas de Apurímac, Perú

${ }^{2}$ Facultad de Medicina Veterinaria y Zootecnia, Universidad Nacional Micaela Bastidas de Apurímac, Perú

${ }^{3}$ Programa de Estudios en Producción Agropecuaria, Instituto de Educación Superior Tecnológico Publico Hermenegildo Miranda Segovia de Antabamba, Perú

${ }^{4}$ E-mail: lcardenas@unamba.edu.pe

Recibido: 21 de junio de 2021

Aceptado para publicación: 30 de septiembre de 2021

Publicado: 22 de diciembre de 2021

CLos autores. Este artículo es publicado por la Rev Inv Vet Perú de la Facultad de Medicina Veterinaria, Universidad Nacional Mayor de San Marcos. Este es un artículo de acceso abierto, distribuido bajo los términos de la licencia Creative Commons Atribución 4.0 Internacional (CC BY 4.0) [https:// creativecommons.org/licenses/by/4.0/deed.es] que permite el uso, distribución y reproducción en cualquier medio, siempre que la obra original sea debidamente citada de su fuente original 
ticia (5.3) fue mayor a los 12 meses de edad de rebrote $(\mathrm{p}<0.05)$ con respecto a los 4 y 8 meses que fueron similares entre sí. El rendimiento de carcasa fue similar (72\%) con la inclusión de 10 y $20 \%$ y disminuyó $(70.6 \%)$ con el $30 \%$ de harina de pisonay $(\mathrm{p}<0.05)$, . La harina de pisonay utilizado como insumo alimenticio para la elaboración de concentrado no afecta las características productivas en cuyes.

Palabras clave: carcasa, consumo, engorde, peso vivo

\section{Abstract}

The aim of this study was to evaluate the effect of three regrowth ages and three levels of inclusion of pisonay (Erythrina edulis) meal on feed consumption and conversion; body weight gain, and carcass rate in guinea pig fattening (Cavia porcellus). Shade-dried leaves and petioles of harvested trees were used and mechanically processed to $2 \mathrm{~mm}$ particle size. In total, 120 improved male guinea pigs were used, distributed in 10 treatments ( 3 regrowth ages, three levels of meal inclusion and a control group with alfalfa meal) for 56 days. The total and daily weight gain, in addition to the feed conversion had a similar response between treatments; feed consumption was lower with the $8 \mathrm{M} 30 \%$ treatment ( $54.0 \mathrm{~g} /$ day $)$ with respect to CA20\% (59.3 g/day) $(\mathrm{p}<0.05)$ and the carcass yield was higher in $4 \mathrm{M} 20 \%(73.5 \%)$ compared to the control diet $(71.1 \%)(\mathrm{p}<0.05)$. Feed consumption $(60.3 \mathrm{~g} /$ day) as feed conversion (5.3) was higher at 12 months of regrowth age $(\mathrm{p}<0.05)$ with respect to 4 and 8 months, which were similar to each other. Carcass yield was similar (72\%) with the inclusion of 10 and $20 \%$ and decreased $(70.6 \%)$ with $30 \%$ pisonay meal $(\mathrm{p}<0.05)$. The pisonay meal used as a feed input for the production of concentrate does not affect the productive characteristics of guinea pigs.

Key words: carcass, consumption, fattening, body weight

\section{INTRODUCCIÓN}

La biomasa cosechada de arbustivas en arreglos silvopastoriles, cuyo contenido nutricional se asemeja a la alfalfa, pueden ser incorporadas a las dietas de cuyes (Cavia porcellus), cubrir los requerimientos nutritivos y reducir la dependencia de los concentrados (Apráez-Guerrero et al., 2013). Los ganaderos reconocen el follaje de especies arbóreas leguminosas como fuente importante de alimento para los animales en época de lluvias (Olivares-Pérez et al., 2011), así como los tallos tiernos de especies arbóreas que se usan para cercas vivas, ramoneo y forraje (Toral-Pérez et al., 2019). La composición nutricional de estas especies varía ampliamen- te en términos de proteína cruda, fibra detergente neutra y digestibilidad de la materia orgánica (León et al., 2012). Este forraje puede proporcionarse a los animales como un suplemento, ya sea mediante corte y acarreo o pastoreo directo (Papanastasis et al., 1998).

La inclusión de forrajes y alimentos no convencionales como harinas en la dieta de cuyes no modifican el comportamiento ni la calidad de la canal (Apraez-Guerrero et al., 2008), ni las características sensoriales de la carne de cuy (Guevara et al., 2014). Además, mediante el proceso de obtención de las harinas se pueden disminuir los factores antinutricionales (Adekojo et al., 2014). El follaje de Erythrina americana se puede usar 
como forraje suplementario para el ganado en la estación crítica por su contenido de proteína cruda (PC: 23.2 y $22.6 \%$ ), fibra detergente neutro (FDN: 52.4 y $58.1 \%$ ) y fibra detergente ácido (FDA: 28.1 y 34.3\%) en época seca y lluvia, respectivamente, que exceden los valores de muchos pastos tropicales comunes cultivados (Ascencio-Rojas et al., 2019), pudiendo ser la única fuente de alimentación en rumiantes menores durante periodos cortos (Hernández-Espinoza et al., 2020).

El follaje de especies arbustivas, cosechado a los 60 días de rebrote, utilizado como suplemento alimenticio en cuyes mestizos no afectaron el comportamiento productivo en comparación a cuyes alimentados con concentrado comercial a voluntad más King grass CT-115 (Reyes-Sánchez et al., 2018). Asimismo, la harina de chachafruto (Erythrina edulis) en sustitución de alimento concentrado comercial en alevines de truchas arcoíris (Oncorhynchus mykiss) (Hernández et al., $2011)$ y la harina de forraje de caraca $(E$. poeppigiana) de 60 días de rebrote como suplemento en dietas para cuyes mostraron buenos resultados (Meza-Bone et al., 2012). En tal sentido, el presente estudio tuvo como objetivo evaluar el efecto de tres edades de rebrote y tres niveles de inclusión de harina de pisonay (Erythrina edulis) sobre el consumo y conversión alimenticia; la ganancia de peso vivo total y diario, y el rendimiento de carcasa en el engorde de cuyes.

\section{Materiales y Métodos}

El trabajo experimental se realizó en un galpón de cuyes con iluminación y ventilación suficiente; ubicada en el sector de Moccospampa, distrito de Tamburco, provincia de Abancay, departamento de Apurímac, Perú. La zona se encuentra a una altitud de $2880 \mathrm{~m}$, con temperatura ambiental entre 6.8 a $23.7^{\circ} \mathrm{C}$, humedad relativa de $73.6 \%$ y precipitación pluvial anual de $1022.5 \mathrm{~mm}$ (según SENAMHI 2019, 2020). Se trabajó con cuyes machos mejorados y destetados de 15 días de edad con pesos vivos uniformes provenientes de una granja de cuyes del distrito de Curahuasi, Abancay. El número de cuyes se determinó por muestreo no probabilístico por conveniencia, siendo de 120 (12 por tratamiento $y$, cada uno con 3 repeticiones de 4 cuyes).

Para la elaboración de la harina se utilizó el follaje, tanto hojas como peciolos de árboles de pisonay (Erythrina edulis) de 4, 8 y 12 meses de rebrote, normalmente utilizados y cosechados para la alimentación de los animales. Se realizó un secado natural bajo sombra y después se procedió a moler el forraje seco a través de un molino de martillo (Maquitaxi, picadora y molino de chala) con zaranda de 2-3 $\mathrm{mm}$.

Se prepararon 10 dietas balanceadas en la empresa Del Corral SRL, Abancay. Para la formulación se consideró los requerimientos nutricionales en condiciones isoproteicas $(17-18 \%)$ e isoenergéticas (3000 kcal de Energía Digestible/kg de MS) (Cuadro 1). La mezcla de los insumos se realizó en una mezcladora horizontal de doble hélice durante 7 min, para luego envasarse en sacos con capacidad de $40 \mathrm{~kg}$. Las dietas se prepararon en cantidad suficiente en una sola oportunidad para todo el experimento y la presentación del alimento terminado fue en harina. El alimento fue ofrecido una vez al día.

Los cuyes fueron alojados en jaulas de alambre tipo aéreo, de $0.9 \times 0.9 \times 0.40 \mathrm{~m}$, que brindaron el confort adecuado a los animales. Las jaulas estaban acondicionadas con comederos tipo tolva y bebederos tipo campana para el consumo de agua fresca a voluntad. El estudio experimental tuvo una duración de 56 días, con una fase previa de acostumbramiento por 7 días.

Los cuyes fueron pesados semanalmente utilizando una balanza digital Henkel BRD04kF ( $\pm 1 \mathrm{~g})$. Se cuantificó la ganancia de peso total y la ganancia diaria a partir de las diferencias de peso vivo inicial y final. El consumo de alimento se determinó por dife- 
Cuadro 1. Composición porcentual y nutricional de las dietas experimentales en base seca

\begin{tabular}{|c|c|c|c|c|c|c|c|c|c|c|}
\hline & \multicolumn{9}{|c|}{ Edad de rebrote de pisonay (meses) } & \multirow{4}{*}{$\begin{array}{l}\text { Dieta } \\
\text { control }\end{array}$} \\
\hline & & 4 & & & 8 & & & 12 & & \\
\hline & \multicolumn{9}{|c|}{ Dietas experimentales: concentración de pisonay, $\%$} & \\
\hline & 10 & 20 & 30 & 10 & 20 & 30 & 10 & 20 & 30 & \\
\hline \multicolumn{11}{|l|}{$\overline{\text { Componentes }}$} \\
\hline Harina de pisonay & 10.0 & 20.0 & 30.0 & 10.0 & 20.0 & 30.0 & 10.0 & 20.0 & 30.0 & \\
\hline Harina de alfalfa & & & & & & & & & & 20.0 \\
\hline Afrecho de trigo & 58.1 & 39.9 & 22.0 & 58.2 & 40.3 & 22.6 & 59.2 & 42.2 & 25.4 & 45.9 \\
\hline Torta de soya & 17.3 & 16.2 & 14.5 & 17.3 & 16.2 & 14.3 & 17.4 & 16.1 & 14.6 & 18.3 \\
\hline Maíz & 11.9 & 21.3 & 31.4 & 11.9 & 21.0 & 31.0 & 10.9 & 19.2 & 27.9 & 11.9 \\
\hline Fosfato dicálcico & & 1.2 & 1.0 & & 1.2 & 1.0 & & 1.2 & 1.0 & 0.9 \\
\hline Carbonato de calcio & 1.6 & 0.4 & & 1.6 & 0.4 & & 1.6 & 0.4 & & 1.4 \\
\hline Sal & 0.5 & 0.5 & 0.5 & 0.5 & 0.5 & 0.5 & 0.5 & 0.5 & 0.5 & 0.6 \\
\hline Vitamina $\mathrm{C}$ & 0.2 & 0.2 & 0.2 & 0.2 & 0.2 & 0.2 & 0.2 & 0.2 & 0.2 & 0.5 \\
\hline Micosecuestrante & 0.1 & 0.1 & 0.1 & 0.1 & 0.1 & 0.1 & 0.1 & 0.1 & 0.1 & 0.2 \\
\hline Premix & 0.1 & 0.1 & 0.1 & 0.1 & 0.1 & 0.1 & 0.1 & 0.1 & 0.1 & 0.1 \\
\hline DL- Metionina & 0.02 & 0.1 & 0.17 & 0.02 & 0.1 & 0.17 & 0.02 & 0.1 & 0.17 & 0.1 \\
\hline \multicolumn{11}{|l|}{ Composición nutricional calculada } \\
\hline Materia seca, $\%$ & 93.7 & 93.6 & 93.5 & 93.6 & 93.6 & 93.5 & 93.6 & 93.6 & 93.8 & 93.4 \\
\hline Proteína, \% MS & 17.9 & 17.7 & 17.5 & 17.8 & 17.7 & 17.6 & 17.9 & 17.8 & 17.8 & 17.4 \\
\hline Energía digestible, Mcal $/ \mathrm{kg}$ & 2.96 & 3.01 & 3.01 & 2.97 & 3.01 & 3.01 & 2.98 & 2.98 & 3.01 & 3.06 \\
\hline
\end{tabular}

rencia del peso del alimento suministrado en el día y el desperdicio registrado al día siguiente, utilizando la balanza indicada previamente. La conversión alimenticia se calculó mediante la división entre el consumo de alimento y la ganancia de peso. También, se determinó el rendimiento de carcasa, que incluyó piel, cabeza, patas y órganos nobles (corazón, hígado y riñón), a través de la división entre el peso de la carcasa y el peso vivo del cuy al beneficio, previo ayuno de 24 horas.

Para el análisis estadístico de los caracteres cuantificables se utilizó el diseño completamente al azar (DCA) con 10 tratamientos (dietas experimentales); y para determinar la diferencia entre los grupos y el control se aplicó la prueba de Dunnett con un nivel de significancia del 0.05. Además, se utilizó un arreglo factorial $3 \times 3$ (tres niveles de edad de rebrote $\mathrm{x}$ tres niveles de inclusión), sin considerar el tratamiento control (dieta control), y. para la comparación de medias de cada factor se utilizó la prueba de Duncan con un nivel de significancia del 0.05.

\section{Resultados y Discusión}

En el Cuadro 2 se presenta la respuesta productiva de los cuyes a los tratamientos experimentales. La inclusión de harina de pisonay no afectó la ganancia de peso vivo ni la conversión alimenticia $(\mathrm{p}>0.05)$. El consumo del alimento propuesto disminuyó con la inclusión del $30 \%$ de harina preparada con rebrotes de 8 meses y el rendimiento de 
Cuadro 2. Comportamiento productivo de cuyes (Cavia porcellus) alimentados con dietas conteniendo tres niveles de harina de pisonay (Erythrina edulis) de tres niveles de edad de rebrote durante ocho semanas a partir de los 21 días de edad

\begin{tabular}{|c|c|c|c|c|c|}
\hline & $\begin{array}{c}\text { Ganancia } \\
\text { de peso } \\
\text { total }(\mathrm{g})\end{array}$ & $\begin{array}{c}\text { Ganancia } \\
\text { de peso } \\
\text { (g/día) }\end{array}$ & $\begin{array}{c}\text { Consumo de } \\
\text { materia seca } \\
\text { (g/día) }\end{array}$ & $\begin{array}{c}\text { Conversión } \\
\text { alimentaria } \\
(\mathrm{g} / \mathrm{g})\end{array}$ & $\begin{array}{c}\text { Rendimiento } \\
\text { de carcasa } \\
(\%)\end{array}$ \\
\hline \multicolumn{6}{|l|}{ Tratamiento $^{1}$} \\
\hline $4 \mathrm{M} 10 \%$ & 687.4 & 12.3 & $57.5^{\mathrm{a}}$ & 4.7 & $71.8^{\mathrm{a}}$ \\
\hline $4 \mathrm{M} 20 \%$ & 658.4 & 11.8 & $55.8^{\mathrm{a}}$ & 4.9 & $73.5^{\mathrm{b}}$ \\
\hline $4 \mathrm{M} 30 \%$ & 646.8 & 11.6 & $57.9^{\mathrm{a}}$ & 5.0 & $71.0^{\mathrm{a}}$ \\
\hline $8 \mathrm{M} 10 \%$ & 697.6 & 12.5 & $56.3^{\mathrm{a}}$ & 4.6 & $72.1^{\mathrm{a}}$ \\
\hline $8 \mathrm{M} 20 \%$ & 661.2 & 11.8 & $59.8^{\mathrm{a}}$ & 5.1 & $71.5^{\mathrm{a}}$ \\
\hline $8 \mathrm{M} 30 \%$ & 620.0 & 11.1 & $54.0^{\mathrm{b}}$ & 5.1 & $69.9^{\mathrm{a}}$ \\
\hline $12 \mathrm{M} 10 \%$ & 645.0 & 11.5 & $59.8^{\mathrm{a}}$ & 5.2 & $71.1^{\mathrm{a}}$ \\
\hline $12 \mathrm{M} 20 \%$ & 625.9 & 11.2 & $59.4^{\mathrm{a}}$ & 5.4 & $72.3^{\mathrm{a}}$ \\
\hline $12 \mathrm{M} 30 \%$ & 663.4 & 11.8 & $61.9^{\mathrm{a}}$ & 5.3 & $71.0^{\mathrm{a}}$ \\
\hline CA $20 \%$ & 621.4 & 11.1 & $59.3^{\mathrm{a}}$ & 5.7 & $71.1^{\mathrm{a}}$ \\
\hline \multicolumn{6}{|l|}{ Factor } \\
\hline \multicolumn{6}{|l|}{ Edad de rebrote } \\
\hline $4 \mathrm{M}$ & 664.2 & 11.6 & $57.0^{\mathrm{b}}$ & $4.9^{\mathrm{b}}$ & 72.1 \\
\hline $8 \mathrm{M}$ & 659.6 & 11.6 & $56.7^{\mathrm{b}}$ & $4.9^{b}$ & 71.1 \\
\hline $12 \mathrm{M}$ & 644.7 & 11.4 & $60.3^{\mathrm{a}}$ & $5.3^{\mathrm{a}}$ & 71.4 \\
\hline \multicolumn{6}{|l|}{ Factor } \\
\hline \multicolumn{6}{|l|}{ Inclusión de harina } \\
\hline $10 \%$ & 676.7 & 11.8 & 57.8 & 4.8 & $71.6^{\mathrm{a}}$ \\
\hline $20 \%$ & 648.5 & 11.5 & 58.3 & 5.1 & $72.4^{\mathrm{a}}$ \\
\hline $30 \%$ & 643.4 & 11.3 & 57.9 & 5.1 & $70.6^{\mathrm{b}}$ \\
\hline \multicolumn{6}{|l|}{ Probabilidad } \\
\hline Tratamientos & 0.6676 & 0.6676 & 0.0001 & 0.2231 & 0.0087 \\
\hline $\begin{array}{l}\text { Edad de rebrote } \\
\text { (E) }\end{array}$ & 0.6861 & 0.6861 & 0.0001 & 0.0441 & 0.1414 \\
\hline $\begin{array}{l}\text { Inclusión de } \\
\text { harina (I) }\end{array}$ & 0.3127 & 0.3127 & 0.7126 & 0.2948 & 0.0012 \\
\hline Ex I & 0.5189 & 0.5189 & 0.0001 & 0.9033 & 0.1951 \\
\hline
\end{tabular}

${ }^{1} \mathrm{M}$ : Meses

carcasa fue mayor con la inclusión de $20 \%$ harina de 4 meses de rebrote al ser comparados con la dieta que incluyó $20 \%$ de harina de alfalfa $(\mathrm{p}<0.05)$. El consumo del alimento, así como la conversión alimenticia fue afec- tado por la mayor edad de rebrote $(\mathrm{p}<0.05)$ y el rendimiento de carcasa disminuyó al incluirse $30 \%$ de harina $(\mathrm{p}<0.05)$. Además, se observó que la interacción edad e inclusión afectó el consumo de materia seca. 
Las especies del género Eryhtrina tiene bondades nutricionales que permiten su utilización en la alimentación de cuyes (Choque et al., 2018; Hernández-Espinoza et al., 2020). Cárdenas-Villanueva et al. (2018) al incluir pisonay como forraje fresco hasta niveles de $50 \%$ en la alimentación de cuyes obtuvieron un mayor peso corporal y mejor rendimiento de la canal, en tanto que Meza et al. (2014a) utilizaron el arbusto tropical $E$. poepigiana como forraje fresco ad libitum en alimentación mixta con concentrado encontraron que fue más eficiente que la alimentación con otras gramíneas. Este mismo forraje sometido a 24 horas de marchitez más un suplemento a base de balanceado comercial permitió obtener un mayor consumo, ganancia de peso, rendimiento a la canal y conversión alimenticia en cuyes (Sánchez et al., 2017).

Por otro lado, la adición de 1 y $2 \%$ de harina de pajuro (E. edulis) en el concentrado para cuyes que recibieron dietas isoproteicas (PC: 20\%) e isoenergéticas (ED: $3.15 \mathrm{Mcal} / \mathrm{kg} \mathrm{MS}$ ) mejoró la conversión alimenticia y el rendimiento de carcasa (Guevara et al., 2013), en tanto que la adición de $20 \%$ de harina de caraca (E. poeppigiana) de 60 días de edad de rebrote en el concentrado (PC: 14-16\%) produjo un mayor consumo de alimento y menor rendimiento de carcasa (Meza et al., 2014마). Estos resultados indican que el uso de alimentos alternativos no convencionales con porcentaje de proteína cruda superior al $20 \%$ y niveles de energía digestible entre 2.9 a $3.0 \mathrm{Mcal} / \mathrm{kg}$ de MS logran mejor desempeño en ganancia de peso, conversión alimenticia y rendimiento de la canal en cuyes (Airahuacho y Vergara, 2017; Macancela-Urdiales et al., 2019).

\section{Conclusiones}

La harina de pisonay (Erythrina edulis) utilizado como insumo alimenticio para la elaboración de concentrado no afecta las características productivas en cuyes (Cavia porcellus).

\section{Agradecimientos}

Por el apoyo económico de la Universidad Nacional Micaela Bastidas de Apurímac para la ejecución del proyecto de investigación, a la familia Sierra Saavedra por su predisposición en habilitar su galpón y permitir la cosecha de los árboles de pisonay y a la empresa Del Corral SRL por su desprendimiento con sus equipos para la elaboración de los alimentos concentrados.

\section{Literatura Citada}

1. Adekojo SA, Adama TZ, Aremu A, Ijaiya AT, Owoleke OE, Ibrahim A.

2014. Effects of dietary inclusion of differently processed Leucaena leucocephala leaf meal on carcass characteristics of rabbits (Oryctolagus cunniculus). Int J Food Sci Nutr Eng 4: 118127. doi: $10.5923 /$ j.food.20140405.02

2. Airahuacho FE, Vergara V. 2017. Evaluation of two levels of digestible energy based on nutritional standards of the NRC (1995) in growth diets for guinea pigs (Cavia porcellus L). Rev Inv Vet Perú 28: 255-264. doi: 10.15381/ rivep.v28i2.13079

3. Apraez-Guerrero JE, FernandezPármo L, Hernandez-Gonzaáes A. 2008. Effect of the usage of grasses and non conventional feed on the productive behavior, carcass performance and meat quality of guinea pigs (Cavia porcellus). Rev Vet Zootec 2: 29-34.

4. Apráez-Guerrero JE, Gómez-Gómez TC, Calpa-Tello JS. 2013. Productive behavior of guinea pigs (Cavia porcellus) under systems with gramineae silvopastoral in mild climate in the department of Nariño, Colombia. Rev Inv Pec 2: 41-48.

5. Ascencio-Rojas L, Valles-de la Mora B, Castillo-Gallegos E, Ibrahim M. 2019. In situ ruminal degradation and effective degradation of foliage from six tree species during dry and rainy seasons 
in Veracruz, Mexico. Agroforestry Systems 93: 123-133. doi: 10.1007/ s10457-018-0184-z

6. Cárdenas-Villanueva LA, SarmientoCasavilca VH, Ramos-Zuniga $R$. 2018. Productive and technological characteristics into guinea pig meat $(\mathrm{Ca}$ via porcellus) using pisonay based-diets (Erythrina sp). J High Andean Res 20: 451-460. doi: 10.18271/ria.2018.422

7. Choque H, Huaita A, Cárdenas LA, Ramos R. 2018. Effect of regrowth age the ruminal degradation of pisonay (Erythrina sp) in Andean valley of Abancay. J High Andean Res 20: 189202. doi: 10.18271/ria.2018.363

8. Guevara J, Díaz P, Bravo N, Vera M, Crisóstomo O, Barbachán H, Huamán D. 2013. Use flour pajuro (Erythrina edulis) as food supplement in guinea pig Lima. Rev Per Quím Ing Quím 16: 21-28.

9. Guevara J, Suca C, Suca F, Barbachan H. 2014. Análisis sensorial de carne de cuyes alimentados con dietas suplementadas con harina de pajuro (Erythrina edulis). Rev Per Quím Ing Quím 17: 59-62.

10. Hernández-Espinoza DF, RamosJuárez JA, González-Garduño R, Lagunes-Espinoza L del C, LópezHerrera MA, Oliva-Hernández J. 2020. Erythrina americana Miller foliage intake in Blackbelly x Pelibuey ewes. Rev Mex Cienc Pecu 11: 70-88. doi: 10.22319/rmcp.v11i1.5226

11. Hernández J, Castillo M, Garay V, Mora A, Caamaño J, Urbina A. 2011. Efecto de la harina de chachafruto (Erythrina edulis triana ex micheli) como suplemento en la alimentación de truchas arco iris (Oncorhynchus mykiss). Agricultura Andina 18: 12-28.

12. León M, Martínez S, Pedraza M, González C. 2012. Chemical composition and in vitro digestibility indicators from fourteen tropical forages. Rev Prod Anim 24(1).
13. Macancela-Urdiales WG, Soca-Pérez M, Sánchez-Santana T. 2019. Productive indicators in Cavia porcellus, fed five forage species in the Austro region of Ecuador. Pastos y Forrajes 42: 262-267.

14. Meza-Bone GA, Sánchez-Laiño AR, Meza-Bone MA, Meza-Bone CJ, Franco-Suescum NG, AvellanedaCevallos JH, Barrera-Alvarez A, et al. 2012. Tropical shrub forage in vivo digestibility for guinea pigs feeding $(\mathrm{Ca}$ via porcellus Linnaeus), in the Ecuadorian littoral. Vet Zootec 6: 8-16.

15. Meza GA, Cabrera RP, Morán JJ, Meza FF, Cabrera AC, Meza CJ, Meza JS, et al. 2014a. Improved fattening guinea pig (Cavia porcellus $\mathrm{L}$ ) based on tropical forage grasses and shrubs in Quevedo, Ecuador. IDESIA 32: 75-80.

16. Meza GA, Loor NJ, Sánchez AR, Avellaneda JH, Meza CJ, Vera DF, Cabanilla MG, et al. 2014b. Leaf meals and tropical shrubby foliage (Morus alba, Erythrina poeppigiana, Tithonia diversifolia and Hibiscus rosasinensis) in feeding guinea pigs (Cavia porcellus Linnaeus). Rev Fac Med Vet Zoot 61: 258-269. doi: 10.15446/ rfmvz.v61n3.46874

17. Olivares-Pérez J, Avilés-Nova F, Albarrán-Portillo B, Rojas-Hernández,S, Castelán-Ortega OA. 2011. Identification, uses and measurement of fodders legumes trees in south farmers of the state of Mexico. Trop Subtrop Agroecosys 14: 739-748.

18. Papanastasis VP, Platis PD, DiniPapanastasi O. 1998. Effects of age and frequency of cutting on productivity of Mediterranean deciduous fodder tree and shrub plantations. Forest Ecol Manag 110: 283-292. doi: 10.1016/S03781127(98)00293-X

19. Reyes-Sánchez N, Vivas J, Aguilar J, Hernández J, Caldera N. 2018. Guinea pigs (Cavia porcellus $\mathrm{L}$ ) supplemen- 
tation with fresh foliage of morera (Morus alba) and Moringa moringa (Moringa oleifera). Rev Cient 18: 7-13. doi: $10.5377 /$ calera.v18i30.7733

20. Sánchez A, Torres E, Espinoza Í, Sánchez J, Sánchez N, Torres B. 2017. Tropical shrub forage in guinea pig fattening (Cavia porcellus
Linnaeus). Rev Amazónica Cien Tecnol 6: 244-249.

21. Toral-Pérez OC, Delgado-Rodríguez M, Gutiérrez-Otero MA, IglesiasGómez JM. 2019. Prospection and collection of arboreal and shrub species in the northeast area of Yaguajay, Cuba. Avances Inv Agropec 23: 55-67. 